\title{
Fourth order Hamiltonian system with some singular nonlinear term and multiplicity result
}

\section{Tacksun Jung ${ }^{1}$ and Q-Heung Choi ${ }^{2^{*}}$ (i)}

\section{"Correspondence:}

qheung@inha.ac.kr

${ }^{2}$ Department of Mathematics

Education, Inha University, Incheon, 402-751, Korea

Full list of author information is

available at the end of the article

\begin{abstract}
We consider a fourth order Hamiltonian system with some singular nonlinear term and multiplicity result. We get two theorems which show the number of weak solutions of this problem. The first theorem is a result which shows that there exists a weak solution for this problem and the second one is an improved result of the first result, which shows that there exist infinitely many weak solutions for this problem. We get the first result by a variational method and critical point theory, and we get the second result by homology theory.
\end{abstract}

MSC: 35Q72

Keywords: fourth order Hamiltonian system; singular nonlinear term; variational method; critical point theory; minimax method; homology theory; (P.S.)c condition

\section{Introduction}

Let $\bar{N}_{\epsilon}(\theta)$ be a closure of an $\epsilon$-neighborhood of $\theta=(0, \ldots, 0), \epsilon>0$ be a fixed small number, and $D$ be an open subset in $R^{n}$ with compact complement $\bar{N}_{\epsilon}(\theta)=R^{n} \backslash D$, $n \geq 2$. Let $c \in R$ and $|\cdot|$ be a norm in $R^{n}$. In this paper we consider the weak solutions $z(t)=\left(z_{1}(t), \ldots, z_{n}(t)\right) \in C^{4}([0,2 \pi], D)$ of a fourth order Hamiltonian system with singular nonlinear term

$$
\begin{aligned}
& \dddot{z}(t)+c \ddot{z}(t)+\operatorname{grad}_{z}\left(\frac{1}{|z(t)|^{2 p}}\right)=0, \quad p \geq 1, \\
& z(0)=z(2 \pi), \quad \ddot{z}(0)=\ddot{z}(2 \pi) .
\end{aligned}
$$

Our problems are characterized as a singular fourth order Hamiltonian system with singularity at $\{z(t)=\theta\}, \theta=(0, \ldots, 0)$. The motivation of this paper is the fourth order elliptic problem with singular potential. We recommend the book [1] for the singular elliptic problems. Many authors considered the fourth order elliptic boundary value problem. In particular, Choi and Jung [2] showed that the problem

$$
\begin{aligned}
& \Delta^{2} u+c \Delta u=b u^{+}+s \quad \text { in } \Omega, \\
& u=0, \quad \Delta u=0 \quad \text { on } \partial \Omega,
\end{aligned}
$$

(c) 2016 Jung and Choi. This article is distributed under the terms of the Creative Commons Attribution 4.0 International License (http://creativecommons.org/licenses/by/4.0/), which permits unrestricted use, distribution, and reproduction in any medium, provided you give appropriate credit to the original author(s) and the source, provide a link to the Creative Commons license, and indicate if changes were made. 
has at least two nontrivial solutions when $c<\lambda_{1}, \lambda_{1}\left(\lambda_{1}-c\right)<b<\lambda_{2}\left(\lambda_{2}-c\right)$, and $s<0$ or when $\lambda_{1}<c<\lambda_{2}, b<\lambda_{1}\left(\lambda_{1}-c\right)$, and $s>0$. We obtained these results by using a variational reduction method. We [3] also proved that when $c<\lambda_{1}, \lambda_{1}\left(\lambda_{1}-c\right)<b<\lambda_{2}\left(\lambda_{2}-c\right)$, and $s<0,(1.2)$ has at least three nontrivial solutions by using degree theory. Tarantello [4] also studied

$$
\begin{aligned}
& \Delta^{2} u+c \Delta u=b\left((u+1)^{+}-1\right), \\
& u=0, \quad \Delta u=0 \quad \text { on } \partial \Omega .
\end{aligned}
$$

She showed that if $c<\lambda_{1}$ and $b \geq \lambda_{1}\left(\lambda_{1}-c\right)$, then (1.3) has a negative solution. She obtained this result by degree theory. Micheletti and Pistoia [5] also proved that if $c<\lambda_{1}$ and $b \geq$ $\lambda_{2}\left(\lambda_{2}-c\right)$ then (1.3) has at least three solutions by the variational linking theorem and Leray-Schauder degree theory.

The eigenvalue problem

$$
\begin{aligned}
& \ddot{u}+\lambda u=0 \quad \text { in }(0,2 \pi), \\
& u(0)=u(2 \pi)=0,
\end{aligned}
$$

has many eigenvalues $\lambda_{j}, j \geq 1$, and corresponding eigenfunctions $\phi_{j}, j \geq 1$, suitably normalized with respect to $L^{2}([0,2 \pi])$ inner product and each eigenvalue $\lambda_{j}$ is repeated as often as its multiplicity. The eigenvalue problem

$$
\begin{aligned}
& \dddot{u}(t)+c \ddot{u}(t)=\mu u \quad \text { in }(0,2 \pi), \\
& u(0)=u(2 \pi)=0, \quad \ddot{u}(0)=\ddot{u}(2 \pi)=0,
\end{aligned}
$$

has also infinitely many eigenvalues $\mu_{j}=\lambda_{j}\left(\lambda_{j}-c\right), j \geq 1$, and corresponding eigenfunctions $\phi_{j}, j \geq 1$. We note that $\mu_{1}<\mu_{2} \leq \mu_{3}, \ldots, \mu_{j} \rightarrow+\infty$.

In this paper we are trying to find the weak solutions $z(t) \in C^{4}([0,2 \pi], D) \cap \Lambda D$ of the system (1.1) satisfying

$$
\int_{0}^{2 \pi}[\ddot{z}(t) \cdot \ddot{\phi}(t)-c \dot{z}(t) \cdot \dot{\phi}(t)] d t+\int_{\Omega} \operatorname{grad}_{u}\left(\frac{1}{|z(t)|^{2 p}}\right) \cdot \phi(t) d t=0
$$

for all $\phi(t) \in C^{4}([0,2 \pi], D) \cap \Lambda D$, where $\Lambda D$ is introduced in Section 2 .

Theorem 1.1 Assume that $\lambda_{j}<c<\lambda_{j+1}, j \geq 1$. Then the system (1.1) has at least one nontrivial weak solution.

Moreover, we improve Theorem 1.1 as follows.

Theorem 1.2 Assume that $\lambda_{j}<c<\lambda_{j+1}, j \geq 1$. Then the system (1.1) has infinitely many nontrivial weak solutions.

For the proof of Theorem 1.1 we follow the approach of the variational method and use a minimax method in critical point theory on the loop space $\Lambda D$, and for the proof of Theorem 1.2 we follow homology theory. In Section 2, we introduce a loop subspace $\Lambda D$ 
of the Banach space, and we prove that the associated functional $J$ of $(1.1)$ satisfies the (P.S.) condition on the loop subspace $\Lambda D$. In Section 3, we use a minimax method and critical point theory for the existence of a nontrivial weak solution of (1.1) and prove Theorem 1.1. We also prove Theorem 1.2 by using critical point theory and homology theory to prove the existence of infinitely many nontrivial weak solutions.

\section{Variational approach}

Let $L^{2}([0,2 \pi], R)$ be a square integrable function space defined on $[0,2 \pi]$. Any element $x$ in $L^{2}([0,2 \pi], R)$ can be written as

$$
x=\sum h_{k} \phi_{k} \quad \text { with } \sum h_{k}^{2}<\infty
$$

We shall denote the subset of $L^{2}([0,2 \pi], R)$ satisfying the $2 \pi$-periodic condition, by $L^{2}\left(S^{1}, R\right)$. Similar notations will be used for other $2 \pi$-periodic function spaces. We define a subspace $W$ of $L^{2}\left(S^{1}, R\right)$ as follows:

$$
W=\left\{x \in L^{2}\left(S^{1}, R\right)\left|\sum\right| \mu_{k} \mid h_{k}^{2}<\infty\right\}
$$

Then this is a complete normed space with a norm

$$
\|x\|_{W}=\left[\sum\left|\mu_{k}\right| h_{k}^{2}\right]^{\frac{1}{2}}
$$

Let

$$
\begin{aligned}
& W^{+}=\left\{x \in W \mid h_{k}=0 \text { if } \mu_{k}<0\right\}, \\
& W^{-}=\left\{x \in W \mid h_{k}=0 \text { if } \mu_{k}>0\right\} .
\end{aligned}
$$

Then $W=W^{-} \oplus W^{+}$, for $x \in W, x=x^{-}+x^{+} \in W^{-} \oplus W^{+}$. Let $E$ be the $n$ Cartesian product space of $W$, i.e.,

$$
E=W \times W \times \cdots \times W .
$$

Let $E^{+}$and $E^{-}$be the subspaces on which the functional

$$
z \mapsto A(z)=\int_{0}^{2 \pi}\left[|\ddot{z}(t)|^{2}-c|\dot{z}(t)|^{2}\right] d t
$$

is positive definite and negative definite, respectively. Then

$$
E=E^{+} \oplus E^{-} .
$$

Let $P^{+}$be the projection from $E$ onto $E^{+}$and $P^{-}$the projection from $E$ onto $E^{-}$. The norm in $E$ is given by

$$
\|z\|_{E}^{2}=\left\|P^{+} z\right\|_{E}^{2}+\left\|P^{-} z\right\|_{E}^{2}
$$


where $\left\|P^{+} z\right\|_{E}^{2}=\sum_{i=1}^{n}\left\|P^{+} z_{i}\right\|_{W}^{2},\left\|P^{-} z\right\|_{E}^{2}=\sum_{i=1}^{n}\left\|P^{-} z_{i}\right\|_{W}^{2}, z=\left(z_{1}, \ldots, z_{n}\right)$. Let $v_{\mu_{i}}^{1}, v_{\mu_{i}}^{2}, \ldots, v_{\mu_{i}}^{n}$ be the eigenvalues of the matrix

$$
\operatorname{det}\left(\mu_{i} I\right)=\left(\begin{array}{ccccc}
\mu_{i} & 0 & 0 & \ldots & 0 \\
0 & \mu_{i} & 0 & \ldots & 0 \\
\vdots & \vdots & \vdots & \ddots & \vdots \\
0 & 0 & 0 & \ldots & \mu_{i}
\end{array}\right) \in M_{n \times n}(R), \quad i=1, \ldots, n
$$

that is,

$$
\nu_{\mu_{i}}^{k}=\mu_{i}, \quad i \geq 1, \text { for all } k=1, \ldots, n
$$

Let $\left(c_{1, \mu_{i}}^{1}, \ldots, c_{n, \mu_{i}}^{1}\right),\left(c_{1, \mu_{i}}^{2}, \ldots, c_{n, \mu_{i}}^{2}\right), \ldots,\left(c_{1, \mu_{i}}^{n}, \ldots, c_{n, \mu_{i}}^{n}\right)$ be the eigenvectors of the matrix

$$
\operatorname{det}\left(\mu_{i} I\right)=\left(\begin{array}{ccccc}
\mu_{i} & 0 & 0 & \ldots & 0 \\
0 & \mu_{i} & 0 & \ldots & 0 \\
\vdots & \vdots & \ddots & \vdots & \vdots \\
0 & 0 & 0 & \ldots & \mu_{i}
\end{array}\right) \in M_{n \times n}(R)
$$

corresponding to the eigenvalues $v_{\mu_{i}}^{1}, v_{\mu_{i}}^{2}, \ldots, v_{\mu_{i}}^{n}$, respectively. Since $v_{\mu_{i}}^{k}=\mu_{i}$ for all $k=$ $1,2, \ldots, n,\left(c_{1, \mu_{i}}^{1}, \ldots, c_{n, \mu_{i}}^{1}\right)=\cdots=\left(c_{1, \mu_{i}}^{n}, \ldots, c_{n, \mu_{i}}^{n}\right)$. Let us set

$$
\left(c_{1, \mu_{i}}, \ldots, c_{n, \mu_{i}}\right)=\left(c_{1, \mu_{i}}^{1}, \ldots, c_{n, \mu_{i}}^{1}\right)=\cdots=\left(c_{1, \mu_{i}}^{n}, \ldots, c_{n, \mu_{i}}^{n}\right) .
$$

Let us set

$$
\begin{aligned}
W_{\mu_{i}} & =\operatorname{span}\left\{\phi_{i} \mid \mu_{j}=\mu_{i}\right\}, \\
E_{\mu_{i}}= & \left\{\left(c_{1, \mu_{i}} \phi, \ldots, c_{n, \mu_{i}} \phi\right) \in E \mid\left(c_{1}, \ldots, c_{n}\right) \in R^{n}, \phi \in W_{\mu_{i}}\right\}, \\
E_{\mu_{i}}^{1}= & \left\{\left(c_{1, \mu_{i}}^{1} \phi, \ldots, c_{n, \mu_{i}}^{1} \phi\right) \in E \mid \phi \in W_{\mu_{i}}\right\}, \\
& \vdots \\
E_{\mu_{i}}^{n}= & \left\{\left(c_{1, \mu_{i}}^{n} \phi, \ldots, c_{n, \mu_{i}}^{n} \phi\right) \in E \mid \phi \in W_{\mu_{i}}\right\} .
\end{aligned}
$$

We note that

$$
E_{\mu_{i}} \equiv E_{\mu_{i}}^{1} \equiv \cdots \equiv E_{\mu_{i}}^{n}
$$

and

$$
E=\bigoplus_{i \geq 1} E_{\mu_{i}}
$$

Let us introduce an open set of the Hilbert space $E$ as follows:

$$
\Lambda D=\left\{z \in E \mid z(t) \in D=R^{n} \backslash \bar{N}_{\epsilon}(\theta), \epsilon>0 \text { is a small number, } \forall t \in S^{1}\right\} .
$$


Let us consider the functional on $\Lambda D$

$$
J(z)=\frac{1}{2} \int_{0}^{2 \pi}\left[|\ddot{z}(t)|^{2}-c|\dot{z}(t)|^{2}\right] d t+\int_{0}^{2 \pi} \frac{1}{|z(t)|^{2 p}} d t, \quad p \geq 1 .
$$

The Euler equation for $J$ is (1.1).

By Lemma 2.1, $J \in C^{1}(\Lambda D, R)$, and so the weak solutions of system (1.1) coincide with the critical points of the associated functional $J(z)$.

Lemma 2.1 J(z) is continuous and Fréchet differentiable in $\Lambda D$ with Fréchet derivative

$$
D J(z) v=\int_{0}^{2 \pi}\left[\ddot{z}(t) \cdot \ddot{w}(t)-c \dot{z}(t) \cdot \dot{w}(t)+\operatorname{grad}_{z} \frac{1}{|z(t)|^{2 p}} \cdot w(t)\right] d x \quad \forall w \in \Lambda D .
$$

Moreover, $D J \in C$. That is, $J \in C^{1}$.

Proof First we prove that $J(z)$ is continuous. For $z, w \in \Lambda D$,

$$
\begin{aligned}
|J(z+w)-J(z)| & \mid \frac{1}{2} \int_{0}^{2 \pi}(z \dddot{+} w+c z \ddot{+} w) \cdot(z+w) d t \\
& \quad+\int_{0}^{2 \pi} \frac{1}{|z(t)+w(t)|^{2 p}} d t \\
& \quad-\frac{1}{2} \int_{0}^{2 \pi}(\dddot{z}+c \ddot{z}) \cdot z d t-\int_{0}^{2 \pi} \frac{1}{|z(t)|^{2 p}} d t \mid \\
=\mid & \frac{1}{2} \int_{0}^{2 \pi}[(\dddot{z}+c \ddot{z}) \cdot w+(\dddot{w}+c \ddot{w}) \cdot z+(\dddot{w}+c \ddot{w}) \cdot w] d t \\
& +\int_{0}^{2 \pi}\left(\frac{1}{|z(t)+w(t)|^{2 p}}-\frac{1}{|z(t)|^{2 p}}\right) d t \mid .
\end{aligned}
$$

We have

$$
\begin{aligned}
& \left|\int_{0}^{2 \pi}\left[\frac{1}{|z(t)+w(t)|^{2 p}}-\frac{1}{|z(t)|^{2 p}}\right] d t\right| \\
& \quad \leq\left|\int_{0}^{2 \pi}\left[\operatorname{grad}_{z} \frac{1}{|z(t)|^{2 p}} \cdot w+O\left(\|w\|_{E}\right)\right] d t\right|=O\left(\|w\|_{E}\right) .
\end{aligned}
$$

Thus we have

$$
|J(z+w)-J(z)|=O\left(\|w\|_{E}\right)
$$

Next we shall prove that $J(z)$ is Fréchet differentiable in $\Lambda D$. For $z, w \in \Lambda D$,

$$
\begin{aligned}
& |J(z+w)-J(z)-D J(z) w| \\
& \quad=\mid \frac{1}{2} \int_{0}^{2 \pi}(z \dddot{+} w+c z \ddot{+} w) \cdot(z+w) d t+\int_{0}^{2 \pi} \frac{1}{|z(t)+w(t)|^{2 p}} d t
\end{aligned}
$$




$$
\begin{aligned}
& -\frac{1}{2} \int_{0}^{2 \pi}(\dddot{z}+c \ddot{z}) \cdot z d t-\int_{0}^{2 \pi} \frac{1}{|z(t)|^{2 p}} d t \\
& -\int_{0}^{2 \pi}\left(\dddot{z}+c \ddot{z}+\operatorname{grad}_{z} \frac{1}{|z(t)|^{2 p}}\right) \cdot w d t \mid \\
= & \mid \frac{1}{2} \int_{0}^{2 \pi}[(\dddot{w}+c \ddot{w}) \cdot z+(\dddot{w}+c \ddot{w}) \cdot w] d t \\
& +\int_{0}^{2 \pi}\left(\frac{1}{|z(t)+w(t)|^{2 p}}-\frac{1}{|z(t)|^{2 p}}\right) d t-\int_{0}^{2 \pi} \operatorname{grad}_{z} \frac{1}{|z(t)|^{2 p}} \cdot w d t \mid .
\end{aligned}
$$

Thus by (2.3), we have

$$
|J(z+w)-J(z)-D J(z) w|=O\left(\|w\|_{E}\right)
$$

Similarly, it is easily checked that $J \in C^{1}$.

Lemma 2.2 Assume that $\lambda_{j}<c<\lambda_{j+1}, j \geq 1$. Let $\left\{z_{k}\right\} \subset \Lambda D, z_{k}(t) \in Z$, and $z_{k} \rightarrow z$ weakly in $\Lambda D$ with $z \in \partial \Lambda D$. Then $J\left(z_{k}\right) \rightarrow \infty$, where $Z$ is a neighborhood of $\theta=(0, \ldots, 0)$.

Proof Since $\frac{1}{z\left(t^{2}\right)^{2 p}}$ has a singular point $\theta=(0, \ldots, 0)$ in $R^{n}$, the conclusion follows.

Now, we shall prove that $J(z)$ satisfies $(P . S .)_{\gamma}$ condition for any $\gamma \in R$.

Lemma 2.3 Assume that $\lambda_{j}<c<\lambda_{j+1}, j \geq 1$. Then if $\left\|z_{k}\right\|_{E} \rightarrow \infty$, then there exist $\left(z_{h_{k}}\right)_{k}$ and $z$ in $\Lambda D$ such that

$$
\operatorname{grad}_{z} \frac{1}{\left|z_{h_{k}}(t)\right|^{2 p}} \rightarrow z \in \Lambda D, \quad \frac{z_{h_{k}}}{\left\|z_{h_{k}}\right\|_{E}} \rightarrow \theta, \quad \theta=(0, \ldots, 0) .
$$

Proof Let $\left\|z_{k}\right\|_{E} \rightarrow \infty$. Then $\frac{1}{\left|z_{k}(t)\right|^{2 p}}$ is bounded, it follows that

$$
\int_{0}^{2 \pi} \frac{1}{\left|z_{k}(t)\right|^{2 p}} d t \rightarrow 0
$$

Since

$$
\int_{0}^{2 \pi} \frac{\left[\operatorname{grad}_{z} \frac{1}{\left|z_{k}(t)\right|^{2 p}} \cdot z_{k}(t)-2 \frac{1}{\left|z_{k}(t)\right|^{2 p}}\right]}{\left\|z_{k}\right\|_{E}} d t \rightarrow 0
$$

by (2.5), we have

$$
\int_{0}^{2 \pi} \frac{\operatorname{grad}_{z} \frac{1}{\left|z_{k}(t)\right|^{2 p}} \cdot z_{k}(t)}{\left\|z_{k}\right\|_{E}} d t \rightarrow 0
$$

Thus the sequence $\left(\int_{0}^{2 \pi} \frac{\operatorname{grad}_{z} \frac{1}{\left|z_{k}(t)\right|^{2 p}} \cdot z_{k}(t)}{\left\|z_{k}\right\|_{E}} d t\right)_{k}$ is bounded. It follows from (2.6) that there exists a subsequence $\left(z_{h_{k}}\right)_{k}$ such that

$$
\lim _{k \rightarrow \infty} \frac{\int_{0}^{2 \pi}\left[\operatorname{grad}_{z} \frac{1}{\left|z_{h_{k}}(t)\right|^{2 p}} \cdot z_{h_{k}}(t)\right] d t}{\left\|z_{h_{k}}\right\|_{E}}=\lim _{k \rightarrow \infty} \int_{0}^{2 \pi} \operatorname{grad}_{z} \frac{1}{\left|z_{h_{k}}(t)\right|^{2 p}} \cdot \frac{z_{h_{k}}(t)}{\left\|z_{h_{k}}\right\|_{E}} d t=0
$$


Since $\operatorname{grad}_{z} \frac{1}{\mid z_{k}(t)^{2 p}}$ is bounded when $\left\|z_{k}\right\|_{E} \rightarrow \infty$, it follows from (2.7) that there exists $z$ in $\Lambda D$ such that

$$
\operatorname{grad}_{z} \frac{1}{\left|z_{h_{k}}(t)\right|^{2 p}} \rightarrow z \in \Lambda D, \quad \frac{z_{h_{k}}}{\left\|z_{h_{k}}\right\|_{E}} \rightarrow \theta .
$$

Thus the lemma is proved.

Lemma 2.4 Assume that $\lambda_{j}<c<\lambda_{j+1}, j \geq 1$. Then $J(z)$ satisfies the $(P . S .)_{\gamma}$ condition for any $\gamma \in R$.

Proof Let $\gamma \in R$ and $\left(z_{k}\right)_{k} \subset \Lambda D$ be a sequence such that $J\left(z_{k}\right) \rightarrow \gamma$ and

$$
D J\left(z_{k}\right)=\dddot{z}_{k}(t)+c \ddot{z_{k}}(t)+\operatorname{grad}_{z}\left(\frac{1}{\left|z_{k}(t)\right|^{2 p}}\right) \longrightarrow \theta, \quad \theta=(0, \ldots, 0) \text { in } \Lambda D
$$

or equivalently

$$
\left\|P_{+} z_{k}(t)\right\|-\left\|P_{-} z_{k}(t)\right\|+\left(D_{t t t t}+c D_{t t}\right)^{-1}\left(\operatorname{grad}_{z}\left(\frac{1}{\left|z_{k}(t)\right|^{2 p}}\right)\right) \rightarrow \theta,
$$

where $D_{t t t t} z_{k}(t)=\dddot{z}_{k}(t),\left(D_{t t t t}+c D_{t t}\right)^{-1}$ is a compact operator. We shall show that $\left(z_{k}\right)_{k}$ has a convergent subsequence. We claim that $\left\{z_{k}\right\}$ is bounded in $\Lambda D$. By contradiction, we suppose that $\left\|z_{k}\right\|_{E} \rightarrow \infty$ and set $w_{k}=\frac{z_{k}}{\left\|z_{k}\right\|_{E}}$. Since $\left(w_{k}\right)_{k}$ is bounded, up to a subsequence, $\left(w_{k}\right)_{k}$ converges weakly to some $w_{0}$ in $\Lambda D$. Since $J\left(z_{k}\right) \rightarrow \gamma$ and $D J\left(z_{k}\right) \rightarrow 0$, we have

$$
\frac{D J\left(z_{k}\right) \cdot\left(z_{k}\right)}{\left\|z_{k}\right\|_{E}}=\frac{2 J\left(z_{k}\right)}{\left\|z_{k}\right\|_{E}}+\frac{\int_{0}^{2 \pi}\left[\operatorname{grad}_{z} \frac{1}{\left|z_{k}(t)\right|^{2 p}} \cdot z_{k}(t)-2 \frac{1}{\left|z_{k}(t)\right|^{2 p}}\right] d t}{\left\|z_{k}\right\|_{E}} \longrightarrow 0 .
$$

Thus we have

$$
\frac{\int_{0}^{2 \pi}\left[\operatorname{grad}_{z} \frac{1}{\left|z_{k}(t)\right|^{2 p}} \cdot z_{k}(t)-2 \frac{1}{\left|z_{k}(t)\right|^{2 p}}\right] d t}{\left\|z_{k}\right\|_{E}} \rightarrow 0 .
$$

By Lemma 2.3 and (2.8), there exist $\left(z_{h_{k}}\right)_{k}$ and $z$ in $\Lambda D$ such that

$$
\operatorname{grad}_{u} \frac{1}{\left|z_{h_{k}}(t)\right|^{2 p}} \rightarrow z \in \Lambda D, \quad \text { and } \quad \frac{z_{h_{k}}}{\left\|z_{h_{k}}\right\|_{E}} \rightarrow \theta
$$

Thus we have $w_{0}=0$, which is absurd because $\left\|w_{0}\right\|_{E}=1$. Thus $\left\{z_{k}\right\}$ is bounded in $\Lambda D$. Thus $\left(z_{k}\right)_{k}$ has a convergent subsequence converging weakly to some $z$ in $\Lambda D$. We claim that this subsequence of $\left(z_{k}\right)_{k}$ converges strongly to $z$. By $D J\left(z_{k}\right) \rightarrow \theta$, we have

$$
D J\left(z_{k}\right)=\dddot{z}_{k}+c \ddot{z}_{k}+\operatorname{grad}_{z} \frac{1}{\left|z_{k}(t)\right|^{2 p}} \longrightarrow \theta .
$$

We claim that the mapping $z_{k} \rightarrow \mapsto\left(\operatorname{grad}_{z} \frac{1}{\left|z_{k}(t)\right|^{2 p}}\right)_{k}$ is compact. Since the embedding $\Lambda D \hookrightarrow C^{2}\left([0,2 \pi] \times \Lambda D, R^{n}\right)$ is compact, the sequence $\left(\int_{0}^{2 \pi}\left[\operatorname{grad}_{z} \frac{1}{\left|z_{k}(t)\right|^{2 p}} \cdot z_{k}(t) d t\right)_{n}\right.$ has a convergent subsequence which converges to $\int_{0}^{2 \pi}\left[\operatorname{grad}_{z} \frac{1}{|z(t)|^{2 p}} \cdot z(t) d t\right.$. Because $\left\{z_{k}\right\}$ is bounded and the subsequence of $\left(z_{k}\right)_{k}$ converges weakly to some $z$ in $\Lambda D,\left(\operatorname{grad}_{z} \frac{1}{\left|z_{k}(t)\right|^{2 p}}\right)_{k}$ is bounded. Since $\left(D_{t t t t}+c D_{t t}\right)^{-1}$ is compact, by $(2.8),\left(P_{+} z_{k}\right)_{k}$ and $\left(P_{-} z_{k}\right)_{k}$ have subsequences 
converging strongly. Thus $\left(z_{k}\right)_{k}$ has a subsequence converging strongly. Thus the lemma is proved.

\section{Proofs of Theorems 1.1 and 1.2}

Lemma 3.1 There exists a sequence of integers

$$
b_{1}<b_{2}<\cdots<b_{i}<\cdots, \quad b_{i} \rightarrow \infty,
$$

such that $H_{b_{i}}(\Lambda D) \neq 0$.

Proof Let $\epsilon>0$ be a fixed small number such that $\bar{N}_{\epsilon}(\theta)$ contains $\theta$, and choose $R>0$ such that $\bar{N}_{\epsilon}(\theta) \subset \operatorname{int}\left(B_{R}\right)$. Then we have

$$
R^{n} \backslash B_{R} \subset D \subset R^{n} \backslash\{\theta\}
$$

Since $R^{n} \backslash B_{R}$ is a deformation retract of $R^{n} \backslash\{\theta\}, \Lambda\left(R^{n} \backslash B_{R}\right)$ is a deformation retract of $\Lambda\left(R^{n} \backslash\{\theta\}\right)$, so $\Lambda\left(R^{n} \backslash B_{R}\right)$ is a deformation retract of $\lambda D$. Then we have

$$
\begin{aligned}
H_{*}(\Lambda D) & \cong H_{*}\left(\Lambda\left(R^{n} \backslash B_{R}\right)\right) \oplus H_{*}\left(\Lambda D, \Lambda\left(R^{n} \backslash B_{R}\right)\right) \\
& \cong H_{*}\left(\Lambda\left(S^{n-1}\right)\right) \oplus H_{*}\left(\Lambda D, \Lambda\left(S^{n-1}\right)\right) .
\end{aligned}
$$

By [6], the Poincaré series of $\Lambda\left(S^{n-1}\right)$ is written as

$$
P_{t}\left(\Lambda\left(S^{n-1}\right)\right)=\left(1+t^{n}\right)+\frac{t^{n-1}}{1-t^{2(n-1)}}\left(1+t^{n}\right)\left(1+t^{n-1}\right)
$$

with $Z_{2}$ coefficients. Thus the lemma is proved.

Let us set a level set

$$
J_{\gamma}=\{z \in \Lambda D \mid J(z) \leq \gamma\}
$$

and

$$
\beta=\left\{[z] \subset \Lambda D \mid z \in \Lambda D, z(t) \text { is a loop on } D, \forall t \in S^{1}\right\} .
$$

Lemma 3.2 Assume that $\lambda_{j}<c<\lambda_{j+1}, j \geq 1$. For each $\gamma>0$, there exists a finite dimensional singular complex $\Omega=\Omega_{\gamma}$ such that the level set $J_{\gamma}$ is deformed into $\Omega$.

Proof Let us choose $z \in J_{\gamma}$. Then $z \in \Lambda D$ and we have

$$
J(z)=\frac{1}{2} \int_{0}^{2 \pi}\left[|\ddot{z}(t)|^{2}-c|\dot{z}(t)|^{2}\right] d t+\int_{0}^{2 \pi} \frac{1}{|z(t)|^{2 p}} d t \leq \gamma .
$$

We note that there exists a constant $R_{0}>0$ such that

$$
\begin{aligned}
& \text { if }(t, z(t)) \in[0,2 \pi] \times R^{n} \backslash B_{R_{0}}, \\
& \text { then } \frac{1}{|z(t)|^{2 p}}<+\infty \text { and }\left|\operatorname{grad}_{z} \frac{1}{|z(t)|^{2 p}}\right|<+\infty
\end{aligned}
$$


We also note that there exists a neighborhood $Z$ of $\bar{N}_{\epsilon}(\theta)$ such that

$$
\frac{1}{|z(t)|^{2 p}} \geq \frac{C}{d^{2}(z, Z)} \quad \text { for }(t, z) \in[0,2 \pi] \times Z
$$

It follows that there exists a constant $\gamma_{0}>0$ such that

$$
\int_{0}^{2 \pi}\left[|\ddot{z}(t)|^{2}-c|\dot{z}(t)|^{2}\right] d t \leq \gamma_{0}
$$

i.e., we have

$$
\|\dot{z}(t)\|_{E}^{2}-c \int_{0}^{2 \pi}|\dot{z}(t)|^{2} d t \leq \gamma_{0}
$$

Since the number of elements of the set $\left\{\lambda_{i}-c \mid \lambda_{i}-c<0\right\}$ is finite and $\lambda_{i}-c \rightarrow \infty$ as $i \in \infty$, there exists a constant $\gamma_{1}>0$ such that

$$
\int_{0}^{2 \pi}|\dot{z}(t)|^{2} d t \leq \gamma_{1}
$$

By Lemma 2.2, there exists $\epsilon_{0}=\epsilon\left(\gamma, \gamma_{1}\right)$ such that

$$
d\left(z, \bar{N}_{\epsilon}(\theta)\right) \geq \epsilon_{0} \quad \forall z \in J_{\gamma}, \forall t \in S^{1} .
$$

Let us choose an integer $M=M_{\gamma}>2 \pi \frac{\gamma_{1}^{\frac{1}{2}}}{\epsilon_{0}}$ and let

$$
t_{i}=\frac{2 \pi i}{M}, \quad i=1,2, \ldots, M
$$

Let us define a broken line

$$
\bar{z}(t)=\left(1-\frac{1}{2 \pi} M\left(t-t_{i-1}\right)\right) z\left(t_{i-1}\right)+\frac{1}{2 \pi} M\left(t-t_{i-1}\right) z\left(t_{i}\right),
$$

$\forall t \in\left[t_{i-1}, t_{i}\right], i=0,1,2, \ldots, M, \forall x \in J_{\gamma}$. Let

$$
\Omega=\left\{\bar{z}(t) \mid z \in J_{\gamma}\right\}
$$

The corresponding $\bar{z} \mapsto\left(z\left(t_{1}\right), z\left(t_{2}\right), \ldots, z\left(t_{M}\right)\right)$ define a homeomorphism between $\Omega$ and a certain open subset of the $M$-fold product $D \times D \times \cdots \times D$. We first claim that $\Omega \subset \Lambda D$. In fact, $\forall z \in J_{\gamma}$, for $t_{2}>t_{1}$, by (3.3), we have

$$
\begin{aligned}
\left\|z\left(t_{2}\right)-z\left(t_{1}\right)\right\|_{R^{n}} & \leq \int_{t_{1}}^{t_{2}}|\dot{z}(t)| d t \\
& \leq\left(\int_{0}^{2 \pi}|\dot{z}(t)|^{2} d t\right)^{\frac{1}{2}}\left|t_{2}-t_{1}\right|^{\frac{1}{2}} \\
& \leq \gamma_{1}^{\frac{1}{2}}\left|t_{2}-t_{1}\right| .
\end{aligned}
$$


Therefore

$$
\begin{aligned}
d\left(\bar{z}(t), \bar{N}_{\epsilon}(\theta)\right) & \geq d\left(z\left(t_{i}\right), \bar{N}_{\epsilon}(\theta)\right)-\left(1-\frac{1}{2 \pi} M\left(t-t_{i-1}\right)\right)\left\|z\left(t_{i}\right)-z\left(t_{i-1}\right)\right\|_{R^{n}} \\
& \geq \epsilon_{0}-2 \pi M^{-1} \gamma_{1}^{\frac{1}{2}}>0
\end{aligned}
$$

$\forall s \in\left[t_{i-1}, t_{i}\right], i=0,1,2, \ldots, M$. We next claim that there exists $v \in C\left([0,1] \times J_{\gamma}, \Lambda D\right)$ such that $v(0, \cdot)=$ id, and $v\left(1, J_{\gamma}\right)=\Omega$. In fact, let us choose $z(t) \in \Lambda D$ and let us define $v$ as follows:

$$
v(s, z)(t)= \begin{cases}z(t) & \text { for } t \geq 2 \pi s, \\ \left(1-\frac{t-t_{i-1}}{2 \pi s-t_{i-1}}\right) z\left(t_{i-1}\right)+\frac{t-t_{i-1}}{2 \pi s-t_{i-1}} z(2 \pi s) & \text { for } t_{i-1}<t<2 \pi s, \\ \bar{z}(t) & \text { for } t \leq t_{i-1} \leq 2 \pi s \leq t_{i} .\end{cases}
$$

Then $v(0, \cdot)=$ id, and $v\left(1, J_{\gamma}\right)=\Omega$. Thus we prove that $J_{\gamma}$ is deformed into $\Omega$ in the loop space $\Lambda D$. Thus the lemma is proved.

Proof of Theorem 1.1 (Existence of a weak solution) We shall show that the functional $J(z)$ has a critical value by the generalized mountain pass theorem. Thus we first shall show that $J(z)$ satisfies the geometric assumptions of the generalized mountain pass theorem.

Let

$$
\Lambda D^{+}=\Lambda D \cap E^{+}, \quad \Lambda D^{-}=\Lambda D \cap E^{-} .
$$

Then

$$
\Lambda D=\Lambda D^{+} \oplus \Lambda D^{-} .
$$

Let $z \in \Lambda D^{+}$. Then we have

$$
\begin{aligned}
J(z) & =\frac{1}{2}\left\|P^{+} z(t)\right\|_{E}^{2}-\frac{1}{2}\left\|P^{-} z(t)\right\|_{E}^{2}+\int_{0}^{2 \pi} \frac{1}{|z(t)|^{2 p}} d t \\
& =\frac{1}{2}\left\|P^{+} z(t)\right\|_{E}^{2}+\int_{0}^{2 \pi} \frac{1}{|z(t)|^{2 p}} d t .
\end{aligned}
$$

Since $\frac{1}{|z(t)|^{2 p}}$ is positive and bounded, if $z \in \Lambda D^{+}$, then there exists a number $r>0$ such that if $z \in \partial B_{r} \cap \Lambda D^{+}$, then $J(z)>0$. Thus $\inf _{z \in \partial B_{r} \cap \Lambda D^{+}} J(z)>0$. We note that by (3.1), there exists $R>R_{0}$ such that

$$
\text { if } \quad(t, z(t)) \in[0,2 \pi] \times R^{n} \backslash B_{R}, \quad \text { then } \frac{1}{|z(t)|^{2 p}}<+\infty \quad \text { and } \quad\left|\operatorname{grad}_{z} \frac{1}{|z(t)|^{2 p}}\right|<+\infty \text {, }
$$

and by (3.2), there exists a neighborhood $Z$ of $\bar{N}_{\epsilon}(\theta)$ such that

$$
\frac{1}{|z(t)|^{2 p}} \geq \frac{C}{d^{2}(z, Z)} \quad \text { for }(t, z) \in[0,2 \pi] \times Z .
$$


Let us choose $e \in B_{1} \cap \Lambda D^{+}$. Let $z \in \Lambda D^{-} \oplus\{\rho e \mid \rho>0\}$. Then $z=x+y, x \in \Lambda D^{-}, y=\rho e$. Then we have

$$
\begin{aligned}
J(z) & =\frac{1}{2}\left\|P^{+} z(t)\right\|_{E}^{2}-\frac{1}{2}\left\|P^{-} z(t)\right\|_{E}^{2}+\int_{0}^{2 \pi} \frac{1}{|z(t)|^{2 p}} d t \\
& =\frac{1}{2} \rho^{2}-\frac{1}{2}\left\|P^{-} x\right\|^{2}+\int_{\Omega} \frac{1}{|x+\rho e|^{2 p}} d t .
\end{aligned}
$$

By (3.1), there exists constant $R_{0}>0$ such that if $(t, z(t)) \in[0,2 \pi] \times R^{n} \backslash B_{R_{0}}$, then $\left|\frac{1}{|z(t)|^{2 p}}\right|<$ $+\infty$ and $\left|\operatorname{grad}_{z} \frac{1}{|z(t)|^{2 p} \mid}\right|<+\infty$. Thus there exist a large number $R>R_{0}$ and a small number $\rho>0$ such that if $z=x+\rho e \in \partial Q=\partial\left(\left(\left(\bar{B}_{R} \cap \Lambda D^{-}\right) \oplus\left\{r e \mid e \in B_{1} \cap \Lambda D^{+}, 0<r<R\right\}\right) \backslash B_{R_{0}}\right)$, then $J(z)<0$. Thus we have $\sup _{z \in \partial Q} J(z)<0$. By Lemma 2.1, $J(z)$ is continuous and Fréchet differentiable in $\Lambda D$ and, moreover, $D J \in C$. By Lemma 2.4, $J(z)$ satisfies the (P.S.) condition. Thus by the generalized mountain pass theorem [7], $J(z)$ possesses a critical value $c>0$, which is characterized as

$$
c=\inf _{h \in \Gamma} \sup _{z \in Q} J(h(z))
$$

where

$$
\Gamma=\{h \in C(\bar{Q}, \Lambda D) \mid h=\mathrm{id} \text { on } \partial Q\} .
$$

Thus (1.1) has at least one nontrivial weak solution. Thus we prove Theorem 1.1.

Proof of Theorem 1.2 (Existence of infinitely many weak nontrivial solutions) By contradiction, we assume that $J(z)$ has only finitely many critical points $z_{1}, z_{2}, \ldots, z_{l}$ such that by the process of the proof of Theorem 1.1, we can obtain $J\left(z_{j}\right)>0,1 \leq j \leq l$. Let us set

$$
K=\left\{z_{1}, z_{2}, \ldots, z_{l}\right\}
$$

We note that $\operatorname{dim} \operatorname{ker}\left(D^{2} J\left(z_{j}\right)\right) \leq 2 n$, for all $j$. Letting

$$
b^{*}>\max \left\{n M_{0}, \operatorname{ind}\left(J, z_{j}\right)+\operatorname{dim} \operatorname{ker}\left(D^{2} J\left(z_{j}\right)\right) \mid 1 \leq j \leq l\right\},
$$

where $M_{\gamma}$ is defined in the proof of Lemma 3.2, and

$$
\tau>\max \left\{0, J\left(z_{j}\right) \mid 1 \leq j \leq l\right\}
$$

we have

$$
C_{b}\left(J, z_{j}\right)=0 \quad \forall b \geq b^{*}, j=1,2, \ldots
$$

and

$$
H_{*}\left(\Lambda D, J_{0}\right)=H_{*}\left(J_{\tau}, J_{0}\right) .
$$


It follows that

$$
H_{b}\left(\Lambda D, J_{0}\right)=0 \quad \forall b>b^{*} .
$$

Since

$$
\begin{aligned}
& i_{*}: H_{b}(\Lambda D) \longrightarrow H_{b}\left(\Lambda D, J_{0}\right) \text { is injective for } b \geq b^{*}, \\
& H_{b}(\Lambda D)=0 \text { for } b \geq b^{*},
\end{aligned}
$$

which is a contradiction to Lemma 3.1. Thus $J(z)$ has infinitely many critical points $z_{j}$, $j=1,2, \ldots$, in $\Lambda D$.

\section{Competing interests}

The authors declare that they have no competing interests.

\section{Authors' contributions}

All authors contributed equally to the manuscript and read and approved the final manuscript.

\section{Author details}

${ }^{1}$ Department of Mathematics, Kunsan National University, Kunsan, 573-701, Korea. ${ }^{2}$ Department of Mathematics

Education, Inha University, Incheon, 402-751, Korea.

\section{Acknowledgements}

This work was supported by Inha University Research Grant.

Received: 12 April 2016 Accepted: 30 June 2016 Published online: 19 July 2016

\section{References}

1. Ghergu, M, Rădulescu, VD: Singular Elliptic Problems. Bifurcation and Asymptotic Analysis. Clarendon Press, Oxford (2008)

2. Choi, QH, Jung, T: Multiplicity results on nonlinear biharmonic operator. Rocky Mt. J. Math. 29(1), 141-164 (1999)

3. Jung, TS, Choi, QH: Multiplicity results on a nonlinear biharmonic equation. Nonlinear Anal. 30(8), 5083-5092 (1997)

4. Tarantello, G: A note on a semilinear elliptic problem. Differ. Integral Equ. 5(3), 561-565 (1992)

5. Micheletti, AM, Pistoia, A: Multiplicity results for a fourth-order semilinear elliptic problem. Nonlinear Anal. 31(7), 895-908 (1998)

6. Bott, R: Nondegenerate critical manifolds. Ann. Math. 60, 248-261 (1954)

7. Rabinowitz, PH: Minimax Methods in Critical Point Theory with Applications to Differential Equations. CBMS. Regional Conf. Ser. Math., vol. 65. Am. Math. Soc., Providence (1986)

\section{Submit your manuscript to a SpringerOpen ${ }^{\circ}$ journal and benefit from:}

- Convenient online submission

Rigorous peer review

- Immediate publication on acceptance

Open access: articles freely available online

- High visibility within the field

- Retaining the copyright to your article 